\title{
Four pillars of Luhmann's analytical apparatus: Applications for communication research
}

\author{
YJ Sohn, Florida Institute of Technology, School of Arts and Communication, United States \\ ysohn@fit.edu
}

\begin{abstract}
By extending Andersen's (2003) propositions, the current paper formalizes Luhmann's four fundamental analytical frameworks and proposes a model that delineates the relations among them. That is, with the form analysis as the base framework, observation analysis is considered the social extension of form analysis as it involves the distinction observer/observed or ego/alter. Differentiation analysis is described as the factual extension of form as it distinguishes a system (this) and everything else (in its environment). Finally, semantic analysis is considered the temporal extension of form analysis as it focuses on the condensation of meaning over time. In addition, to overcome the abstractness of descriptions in the existing literature, this paper suggests the workable definitions that operationalize the analytical frames. Rich research examples are also presented to demonstrate the broad applicability of the four frameworks in communication research and their analytical gains. These theory-driven analytical frameworks are expected to provide meaningful connections between empirical data and theories, thereby enriching the field of communication research. In turn, more empirical applications will contribute to Luhmann's systems theory by bringing in productive insights.
\end{abstract}

\section{Keywords}

Niklas Luhmann, systems theory, communication research, analytical frameworks, form analysis, observation analysis, differentiation analysis, semantic analysis

\section{Introduction}

Luhmann is undoubtedly one of the most original and controversial theorists in the late $20^{\text {th }}$ century. His innovative insights have challenged the old continent's thinking and provided productive reservoirs of inspiration for countless researchers. Nevertheless, his theory of social systems seems to remain within the closed circle of avid followers and has been completely neglected by outsiders, especially in the Anglophone academic scene. ${ }^{1}$ Many scholars argue that, despite his status as one of the dominant theorists in Europe, Luhmann has been unfairly undervalued in the Anglophone world (see, for instance, Andersen \& Stenner, 2020; Bergthaller \& Schinko, 2011; Borch, 2011; Roth, 2011). The underutilization of Luhmann's theory can be partly explained by the criticism

1 For the polarized acceptance of Luhmann's systems theory in the Anglophone academia, see Arnoldi (2001), and Roth (2011). of its limited research applications. To his critics, Luhmann's highly abstract macro-perspective lacks effective operationalization, thus is non-conducive for empirical scientific research (Baralou, Wolf, \& Meissner, 2012; Johnson \& Leydesdorff, 2015; Šubrt, 2019).

Contrary to this claim, some argue that the methodological aspect is at the center of Luhmann's theory (e.g., Besio \& Pronzini, 2011; Borch, 2011; Roth, 2014b; Roth, Melkonyan, Kaivo-Oja, Manke, \& Dana, 2018). For the intention of the systems theory is to use the theory as a method of discovery that identifies the complex forms which generate and process information within a system (Luhmann, 1997/2012, p. 13; Roth, 2014b, p. 41). Therefore, Brier, Baecker, and Thyssen (2007, p. 9) rightfully argued that for Luhmann there is no distinction between developing a theory and applying it because his theory itself is an applied research of describing the society. In this vein, Roth et al. (2018) described 
Luhmann's theory of systems as a theory-method. By leaning on Merton (1959), the authors contend that "the quality of such a theory-method is consequently not in its robustness against falsification" but "in the scale and scope of scientific problems this theory allows to generate" (Roth et al., 2018, pp. 584-585).

Indeed, Luhmann's theory has offered epistemologically solid perspectives of seeing the world, while practically guiding research for describing and theorizing society. According to a recent quantitative content analysis of Luhmannian research published in English-language academic journals, $62.3 \%$ of the articles printed in the 2010s adopted Luhmann's theory as guiding frameworks, thus demonstrating the instrumental potential for the theory that can lead to fruitful research streams (Sohn, 2020). However, his theoretical approaches have been adopted by researchers under the umbrella term of systems theory without proper labeling or discernment, mainly due to the lack of literature that systematically classifies the full gamut of Luhmann's theoretical-methodological constructs. An exception is Andersen's work (2003), which tackled this issue by putting forth Luhmann's five analytical strategies - form, system, differentiation, semantic, and media analyses. Also, noting the connections among these strategies, he called for a systematic reading of the links among the different analyses (Andersen, 2003, p. 88).

Against this backdrop, by extending Andersen's (2003) study, the current paper undertakes three research tasks: first, it explores Luhmann's fundamental analytical frameworks and the relationships among them, therefore developing a conceptual model linking the frameworks; second, it presents workable definitions of the frameworks, which can provide practical guidance for communication research; and third, it demonstrates the analytical gains when employing them. With these efforts, this paper will provide valuable resources for researchers searching for robust discourse frames or theory-driven frameworks for empirical research.

\section{Luhmann's four fundamental analytical apparatus}

Luhmann's systems theory starts with forms of distinction that distinguishes a system from its environment, and observations that enable describing how social systems reduce complexities of the environment and differentiate subsystems. And all operations of complexity reduction in social systems are based on meaning. Accordingly, this paper proposes Luhmann's four fundamental analytical frameworks: form analysis, observation analysis, differentiation analysis, and semantic analysis. These four frameworks are selected since they serve as foundations for other frames in Luhmann's analysis. For instance, a study that adopts the system-analytical strategy or media analysis in Andersen's (2003) list either implicitly presupposes or explicitly utilizes one or more of these four basic frameworks. ${ }^{2}$ Another point breaking with Andersen (2003) is the inclusion of the observation analysis as one of the basic frameworks. Andersen (2003) discusses "point of observations" as a part of the differentiation framework. However, in addition to providing a reference point for other analyses, observation analysis has its unique functions, as demonstrated in the following discussion.

\subsection{Form analysis}

Form of distinction. In his magnum opus, Die Gesellschaft der Gesellschaft, Luhmann (1997/2012, p. 28) argued to speak of not objects but distinctions. Without distinctions, we would not be able to indicate or observe anything. Hence, Luhmann (2002/2013, p. 49) stressed, "draw a distinction, otherwise nothing will happen at all". Here, a distinction is considered a boundary or "the marking of a difference" (Luhmann, 2002/2013, p. 49). As such, the term distinction is indispensable in understanding Luhmann's conceptual architecture. Luhmann further refined this concept based on the concept of form by drawing on Spencer-Brown (1969). Luh-

2 The "system analytical strategy" is based on the analyses of form, differentiation, and observation, while the "medium strategy" involves the form and differentiation analyses. 
mann defined a form as a distinction that has two sides (Luhmann, 1997/2013, p. 10; $2002 / 2013$, p. 51). Here, a form is not an ontological representation, but the form of distinction that enables something to exist. To illustrate, drawing a circle on a plane creates two divided spaces (i.e., the inside and outside of the circle), the circle itself, and the whole space that includes all. This illustration implies that even space itself is distinguishable only after a distinction has been implemented. Therefore, a form is a triggering moment that generates all (Lee, 2013, p. 43). In the same vein, Luhmann (2002/2013, p. 49) stated,

evidently, creation is nothing but the injunction "Draw a distinction!" Heaven and earth are thereby distinguished, then man, and finally Eve. Creation is thus the imposition of a mode of distinguishing, if God himself is beyond all distinction.

Only after a form is created can one distinguish and mark one side of the divided spaces. An implication is that a form contains two components: distinction (marked by a vertical line) and indication (marked by a horizontal line) (see Figure 1). We can observe things only by indicating one side, not the other, within the frame of distinction. This formula shows the asymmetry of distinction, which indicates the inside of the distinction as the marked state $(m)$, leaving the outside as unmarked state $(n)$. Drawing a distinction imposes an indication of a marked side. In this formula of form, we can cross the distinction from one side to the other with a deferred time, but not simultaneously indicate both sides (Luhmann, 1997/2012).

\section{Figure 1: A form of distinction}

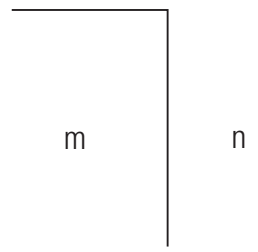

Therefore, we can oscillate focus between the two sides, but never see the unity of the distinction between the two states. To see this unity, we require another level of reflection.

Here comes the concept of re-entry. Re-entry means a distinction copied into the distinction itself or, "the re-entering of the form into the form or of the distinction into the distinguished" (Luhmann, $2002 / 2013$, p. 54). The first distinction is what is actually used, and the re-entered distinction is what is reflected on (see Figure 2). The distinction of a form can have a chain of re-entries.

Figure 2: A form of re-entry

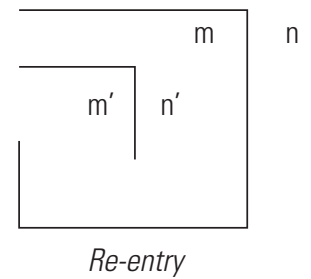

To illustrate, let's call a marked state of a distinction health. While the content of health is indicated, the other side (i.e., the unmarked state) remains unknown, thus forming the state of non-health (i.e., something that is not "health"). The operation of re-entry copies the distinction health/non-health into the context of health. Then, the distinction health / nonhealth along with the content of nonhealth can be scrutinized in the context of health. This reflection reveals a representation of the distinction health/illness. In this example, the content of non-health (the original unmarked state) is not the same as that of illness (the reflected unmarked state), and the initial distinction health/non-health differs from the reflected distinction health / illness.

Form analysis. Form analysis takes the central position of all analyses since it is the starting point of all the guiding frameworks. Andersen (2003, p. 78) described form analysis as "the analysis of the conditions of communication given a specific difference, the guiding distinction of form 
analysis being unity/difference". It implies that investigating the distinction itself (i. e., the unity of distinction) as a blind spot reveals the conditions that enable the communication. In more workable terms, this paper suggests that form analysis involves (a) examining a distinction that makes a difference between two values, and (b) tracking the re-entries of the distinction. Then this form analysis allows understanding the nature of specific communication. For instance, we can answer the question of what "health communication" is by identifying the form health / illness. Once one side of the binary distinction is indicated, another distinction can be drawn within this selected side - i.e., the re-entry of distinction. That is, we indicate the health side, and make a further distinction in this side, distinguishing physical and mental health. This analysis of re-entry can continue multiple times. ${ }^{3}$

In addition, as a form has two sides by definition, indicating the marked side always carries the other unmarked side as the context (Baecker, 2006, p. 124). For instance, indicating "men" presumes a distinction and is meaningful only with the unmarked side such as "women" or "animals". Here, "women" or "animals" not only are the counter-concepts of men but also provide the context in which "men" is interpreted. Forms, therefore, appear to have the communicative structure of concept/counter-concept or concept/context. Hence, we can capture the underlying meaning of communication by identifying the binary distinction concept/counter-concept or depict the framework of communication by examining the concept / context form. For instance, if a term frequently appears in the news media, we can use form analysis to investigate the counter-concept that is ignored in the media reports and thereby locate the unity of the distinction, which will, in turn, reveal the latent or hidden framing that the media reports play with.

The discussions above lead to a working definition of form analysis: the analysis

3 Likewise, we can start with the illness side and continue making further distinctions to reveal what constitutes the concept of illness. of the unity of binary distinctions and their re-entries for identifying the nature of social constructs underlying communication and the conditions of their interpretations by using the scheme of concept/counterconcept or concept/context.

Applications of form analysis. There are several ways of applying form analysis in communication research. First, form analysis is useful for defining concepts or capturing the underlying meanings. For instance, Seidl (2007) used the distinction knowledge / nonknowledge to define intelligence. That is, the author considers intelligence as the re-entry of nonknowledge into knowledge, thus conceptualizing intelligence as the ability to deal with nonknowledge. In this sense, the intelligence of an organization is defined as "the extent that it is aware of its nonknowledge and takes account of this nonknowledge in its operations" (p. 16). In addition, form analysis is a useful tool for detecting and overcoming false distinctions. Spencer-Brown (1969, p. 1) stated that "distinction is perfect continence". This means that a distinction between the concept/ counter-concept should be both jointly exhaustive and mutually exclusive (Roth, Valentinov, \& Clausen, 2020, p. 682). For instance, man vs. nature is false because the two poles are not mutually exclusive (Roth et al., 2020, p. 682). In the same vein, Luhmann (2005) rejected the distinction security / risk since security cannot operate as the counter-concept to risk. Instead, he suggests the distinction risk / danger for analysis. ${ }^{4}$

Second, form analysis can be adopted for detecting emerging communication themes. Gregory, Gibson, and Robinson (2005) explored the core distinctions underlying the participants' communications in the oral health context. In this study, they examined categories emerging

4 Boholm (2008), for instance, examined the communication at public consultation meetings about environmental risk by using the distinction risk/hazard (or risk/danger). The author revealed that, while decision-makers perceived the negative consequences as risks, those affected by a decision (i. e., stakeholders) took them as hazards (or danger). 
Figure 3: The form of the firm depicting the contexts where the operations of a firm occur

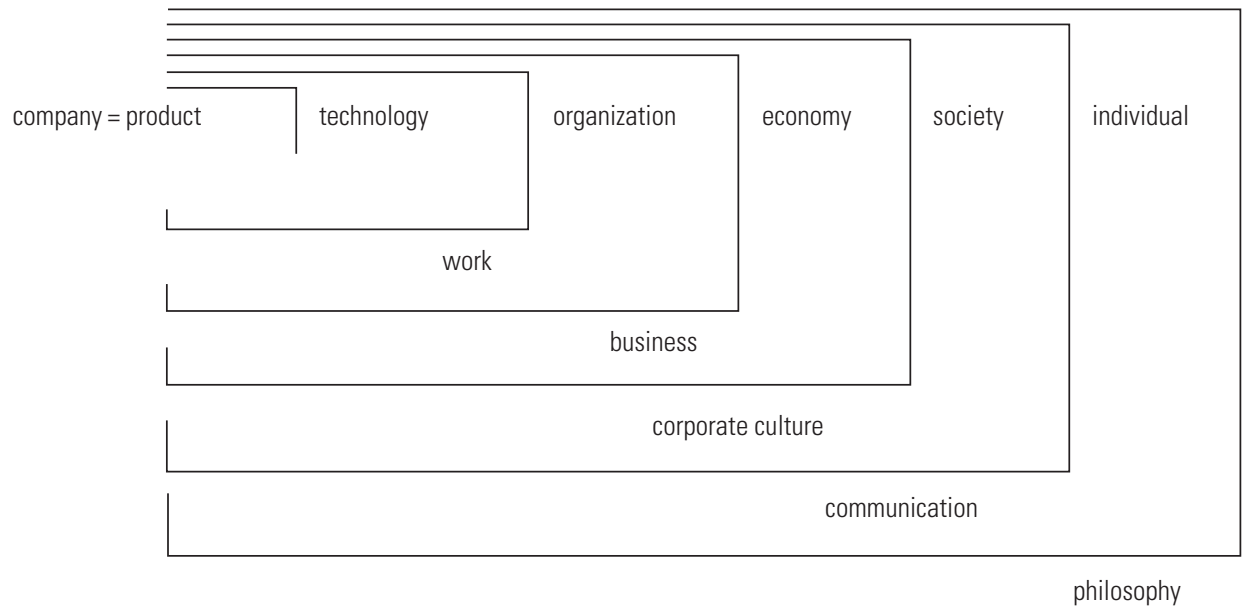

Source: Baecker, 2006, p. 127.

in dentist-patient conversations, searched for the opposing positions (i.e., counter-concepts), and relabeled the distinction unities by connecting the positive/ negative pairs. As a result, six emerging dimensions of the meaning of oral health were identified. $^{5}$

Third, form analysis can facilitate the sensemaking of specific concepts by articulating the contexts in which the concepts are communicated. Baecker (2006), for instance, presented a model that analyzes the structure of organizations based on six distinctions of concept/context and five levels of re-entries: work, business, corporate culture, communication, and philosophy (see Figure 3). This model offers a new perspective for understanding firms' structures and the broader contexts in which their communicative operations occur. Researchers can utilize this kind of form analysis using a chain of re-entries of

5 The emerging categories are positioning of the norm (health / disease); positioning of attribution (internal/ external); positioning of dentistry (trust / distrust); positioning of accessibility (choice / no choice); positioning of commodity (embracing / rejecting); positioning of authenticity (natural / unnatural); positioning of character (admiring / denigrating). form to locate the context against which meaning makes sense or to identify factors that affect communication phenomena. For example, analyzing health organizations with a chain of re-entries will help to understand the contexts and elements for health communication.

\subsection{Observation analysis}

Observation. Based on Spencer-Brown's form calculus, Luhmann unfolded his discourse on observation, the concept that involves distinction and indication (Luhmann, 1997/2012, p. 34). Every observation is tied to the selected distinction. For example, if we observe with the distinction legal / illegal, the potential for the indication is either legal or illegal - and nothing else (Kneer \& Nassehi, 2008, p. 134). While an observation involves indicating one or the other side of a distinction, the distinction itself - i.e., the unity of distinction that simultaneously separates two sides and holds both sides together - remains hidden as long as it is used for observation. The distinction is the "excluded middle that cannot be observed" (Luhmann, $1997 / 2012$, p. 29) and serves as the "invisible condition of seeing, as blind spot" (p. 35, emphasis added). For instance, if we attempt to indicate the distinction le- 
gal/illegal for an observation that uses this distinction, we fall into a paradox of determining whether the distinction legal/ illegal itself is legal or illegal. In a sense, the distinction itself is like perspective: "the perspective is not seen by the one who sees by means of it" (Luhmann, 2002/2013, p. 113).

One way of addressing this blind spot issue is re-entry as described above. The re-entry can be also applied to the distinction observer / observed. For an observer to perform observation, the distinction observer/observed should be hidden to the observer as a blind spot and the condition of observation. The observer can reflect on this distinction by copying the distinction observer / observed into the observer side to reflect on the observed. However, this re-entry causes a paradox of self-inclusion as the observer becomes the object of his own observation. This status is like a Cretan saying, "all Cretans are liars". Therefore, to de-paradoxify, the observer should distinguish self- and other-observations. Self-observation occurs when the observation indicates the reflected observer, while other-observation indicates the reflected observed. ${ }^{6}$

Nevertheless, the re-entering of the distinction observer/observed into the observer side does not allow the observer to see the original distinction. Nor is the original observer the same as the observer reflected by the self-observation. The initial distinction can be observed only by another observer - that is, a second-order observer. The second-order observation means the observation of an observer (Luhmann, 2002/2013, p. 111). A second-order observer can see what is observed by the first-order observer with what distinction, along with what the first-order observer cannot see. While the

6 To illustrate, imagine two persons A and B. At first, A sees that B differs from him. To compare with $\mathrm{B}, \mathrm{A}$ chooses the distinction successful/non-successful. Based on this distinction (i.e., the re-entered distinction), A constructs an image of B (i. e., other-observation) as a successful person, as well as that of himself (i.e., self-observation) as an ordinary person. second-order observation can observe the blind spots of the first-order observation, it is also tied to its distinction, thus having its own blind spots of observation. In this sense, the second-order observation is also the first-order observation.

Observation analysis. Observation analysis has two dimensions: observation based on the distinction self-/other-reference and that based on the distinction first-/second-order observation. The distinction self- / other-reference is related to the re-entry of the distinction observer/ observed into the observer side. Self-reference is the observation of the observers themselves, while other-reference is the observation of the observed other than the observers. Here, self-reference is the observers' self-reflection in which they ask with which specific distinction they observe and what the consequence of the selection has on other-observation. In this sense, the analysis of self- / other-reference becomes the point-of-reference analysis. For instance, researchers can reflect on with which reference points they observe and how their observations are affected by switching their reference points.

Meanwhile, by drawing on SpencerBrown's form calculus, Luhmann described re-entry as "a boundary operation of a calculation which remains at the level of first-order observation and within the context of binary distinctions" (Luhmann, $1996 / 2000$, p. 10). In this sense, self-/ other-references constitute the first-order observation, which, in turn, is analyzed through the second-order observation concerning the observation of an observer. The primary difference between the first- and second-order observation is that the first-order observation is the observation of things or target objects. In contrast, the second-order observation is the observation of observations. More formally, the first-order observation concerns using a specific distinction, whereas the second-order observation concerns making that distinction the object of observation (Bette, 2015, p. 61). Therefore, first-order observers usually ask "what-questions", whereas second-order observers ask "how-questions" about the way in which 
the first-order observer sees the world. For instance, the second-order observation in the disaster communication context can offer new insights by shedding light on "who defines what, when, how, in which context, and with what consequences in the processes and dynamics of disaster-related communication" (Egner, Schorch, Hitzler, Bergmann, \& Wulf, 2012, p. 250). Besides, the second-order observers can see what the first-order observers cannot see - i.e., the specific distinctions that they use for observation and the unindicated content or states. In this vein, the second-order observation can be a powerful tool that enables the reflection of the structures and processes of the first-order observations and questions what remains hidden and non-transparent in the processes (Bette, 2015, pp. 60-61).

By combining the two dimensions, observation analysis can be defined as the analysis of the points of reference and the consequences of their selections based on the guiding distinction observer/observed, which includes the distinction self-reference/other-reference and the distinction second-order/first-order observation.

Applications of observation analysis. As described above, observation analysis is often adopted to observe the difference between the first- and second-order ones. In a study of environmental risks, Boholm (2008) distinguished the first-order observers who were directly affected and the second-order observers who were regulators and decision-makers of the public project and examined the different perceptions of risks between them. The analysis can also be used to reflect on how a choice of reference point affects observation. Kiisel and Vihalemm (2014), for instance, analyzed personal interpretations of warning messages by comparing the direct approach (i. e., first-order observation) with the reflective approach (i.e., second-order observation) and examined how this difference in the level of observation explained the variations in the personal sense of risk, which in turn shaped the reception of a warning message. The authors found that the first-order or direct observers treated the situation given as taken-for-granted and asked how dangerous it was and what they should do. On the contrary, the second-order observers ${ }^{7}$ asked how adequate their construction of the situation was and whether they could trust themselves in trusting the message. This study demonstrates that second-order observation involves evaluating the message along with the messenger, and an awareness of the self as an observer, while first-order observation can avoid time-consuming complexity by accepting the message as trustworthy (Kiisel \& Vihalemm, 2014, p. 278).

Observation analysis is also utilized to offer practical directions for organizational communication. For instance, Andersen and Born (2007) examined the construction of corporate messages by observing how organizations describe themselves. The authors showed that a firm utilizes multiple levels of observation to describe its identity, which emerges differently depending on the position from which it is articulated. They found that organizations communicate established identities, frameworks and operations in the first-order level (e.g., "We at Lego"); claims to control, modify, and / or change organizational principles of identity and operation in the second-order level (e.g., "Lego is (no longer) a force for innovation"); and claim awareness and influence over the reflexivity in the third-order level (e.g., "Lego has had to understand when to support democracy and participation") (Andersen \& Born, 2007, p. 183).

As such, research that adopts observation analysis shows the necessity of more complex considerations in message constructions since both the message creators and recipients are not one-dimensional and more complicated. In this sense, observation analysis provides a useful device for identifying conditions that influence the perceptions of observers and, thus, understanding how messages are created, presented, and interpreted in specific ways. Therefore, observation analysis can

7 In this study, the second-order observers were directed to consciously consider the aims of different messages and accounts of an event. 
be applied to build strategies for message construction and delivery, diagnose and resolve conflicts, and provide other practical recommendations for more effective communication.

\subsection{Differentiation analysis}

Differentiation. In Luhmann's theoretical architecture, the concept of the observer is defined entirely formally with the concepts of distinction and indication (Luhmann, 2002/2013, p. 105). Anything that can distinguish and indicate can be an observer. Not only human consciousness, but also any physical, social, and technical process can observe. ${ }^{8}$ Even the theory of social systems can observe. In this context, Luhmann's social systems theory is reformulated as a "theory that observes reality using a specific distinction, namely the system / environment distinction" (Sciulli, 1994, p. 38).

A system is here defined as a form with two sides (Luhmann, 2002/2013, p. 52), where a system is indicated and the environment remains outside. All systems physical, psychological, or social - constitute and maintain themselves by creating and maintaining differences from their environments (Luhmann, 1984/1995, p. 17). For instance, if the immune system fails to recognize the difference between its elements and foreign substances, it will no longer exist. To sustain the boundary, systems should be operationally closed and autopoietic. An autopoietic system means that it (re)produces itself without any external inputs. Operational closure means that a system's operations are connected with its own operations, but not with those of any other system (Moeller, 2012, pp. 56$57)$. A system has only one type of operation to (re)produce the difference between itself and its environment. As for social systems, the mode of operation is communication, while that of psychic systems is consciousness. Thus, as for social systems,

8 To illustrate, thermostats observe room temperatures to keep them stable; bio cells observe the environments to protect themselves from potential threats, and the mass media system observes society to continue producing information. it is critical to continuously connect communication to further communication for their perpetuations. As such,

the difference between system and environment as a guiding distinction is the point of departure for system-theoretical analysis. (Luhmann, 1984/1995, p. 16)

A critical function of an autopoietic, operationally closed system is reducing the complexity of its environment. Here, complexity refers to a state where a system has more than one possibility of connecting its elements for its continuation (Kneer \& Nassehi, 2008, p. 150). If a system fails in this function due to complexity overload, it cannot distinguish itself from its environment, and eventually vanishes. In the course of reducing the complexity of the environment, however, a system necessarily increases internal complexity. To analogously compare, a system is like a board game, which starts with simple rules to imitate the real world, but soon develops its own various rules and strategies as the game goes on. Thus, the system eventually reaches a point where it needs to differentiate itself internally to cope with the complexity overload. As a result, the differentiation creates subsystems having their own distinctions within the system. In this context, system differentiation is "nothing more than the repetition of system formation within systems" (Luhmann, $1984 / 1995$, p. 18). For instance, the functionally differentiated subsystems of modern society, such as economic, political, and legal systems are the result of the differentiation of the societal system. ${ }^{9}$ Here, each subsystem becomes the environment of other subsystems. Luhmann's theory of social systems is thereby the response to the questions of how a society deals with complexity and how it is internally differentiated into subsystems (Kneer \& Nassehi, 2008, p. 151).

Differentiation analysis. All social systems such as societal function systems,

9 Roth and Schütz (2015) identify ten function systems of modern society: politics, economy, science, art, religion, legal, health, sport, education, and mass media systems. 
organizations, and interaction systems are observing systems that distinguish themselves from their environment through the distinction system / environment and describe themselves with the distinctions. Differentiation analysis then can be broadly defined as the analysis of the distinction that distinguishes a system from its environment. Andersen (2003, p. 82) suggested the difference between similarity and dissimilarity as the guiding distinction of difference analysis. That is, differentiation analysis examines the "similarity of the difference between system and environment in social systems". In this sense, differentiation analysis is considered an analysis of the boundary conditions that demarcate a system and its environment.

The differentiation of social systems entails creating distinct communication forms of different perspectives (Andersen, 2003, p. 82). It is a natural consequence as social systems have communication as their operational mode. Differentiation analysis then involves the analysis of communication forms that a system uses for its observation and self-description. And the analysis of communication forms in modern society involves examining the codes and programs of the societal function systems such as economy, politics, and law. A code is a binary steering distinction of a societal function system. Communication of function systems is structured through the binary code, which marks the boundary of each function system (Luhmann, $1986 / 1989$, p. 36). The code further develops the symbolically generalized communication medium. For instance, the legal system is centered on the "norm" medium based on the code of legal / illegal, while the economic system is geared toward "money" medium based on the code of payment / non-payment. The invariant code is then matched by a plurality of programs. That is, codes and programs form a two-stage selection, where codes are pertinent to selecting the particular item of information, programs are to the selection of the field of selection (Luhmann, 1996/2000, p. 18). As a rule of allocating code values, the variant program allows all the various system-relevant val- ues excluded from the invariant code to be considered part of the system's communication (Baxter, 2013, p. 171; Luhmann, 1997/2012, p. 217). ${ }^{10}$

In summary, this leads to the following working definition of differentiation analysis: the analysis and its application of the boundary conditions distinguishing a system from its environment by using communication forms such as steering binary codes, medium, and/or variant programs utilized in the system. The "application" is added to the definition to emphasize the practical characteristics of differentiation analysis. Indeed, differentiation analysis should be considered to have two levels: On the first level, differentiation analysis concerns examining the differentiation phenomena and the communication forms accompanied by the differentiations; and the second level involves applying the communication forms as the framing of communication in research.

Applications of differentiation analysis. Differentiation analysis in communication research is adopted to explore and compare the communication forms or perspectives utilized in different function systems. For instance, Brier's (2006) case study of "good science gone bad" analyzes the Lomborg case of environmental conflicts in the Agora of the mass media. The study shows how Lomborg used scientific results (allegedly lacking scientific rigorousness) in public debates by switching the frames from truth-based science to economic and political frames. In this study, differentiation analysis reveals a weakness of public knowledge resulting from irreconcilable frames of different systems. In a similar vein, Nobles and Schiff (2004) investigated a legal case that shows how and why legal and medical communications represented in the media select specific information by linking the systems' respective conditioning codes and programs. The authors argued that the legal communication represented by the media is not adequately described as simplifications or distortions of legal reality,

10 For the detailed descriptions of codes, programs, and mediums of the modern function systems, refer to Roth and Schütz (2015). 
just as the legal system unavoidably fails to represent the meaning that medical communication has within its system (Nobles \& Schiff, 2004, p. 221).

As such, differentiation analysis provides a useful tool for media studies, especially for exploring media frames or representations and how the media thematize an issue. Görke (2001) and Görke and Ruhrmann (2003) compared journalism and entertainment as separate systems in terms of the function, code, and programs, and contrasted their discrete roles in the construction of generic risk in public communication. The authors depicted that journalism selects events as actual (of the actuality / potentiality distinction), whereas entertainment selects the reference side (i.e., potentiality side) or the horizon of contingency, thereby providing the public with alternatives for action. Therefore, the authors argued that both journalism and entertainment are an integral part of public communication.

Differentiation analysis can also offer a practical scheme for media discourse analysis or content analysis by providing communication frames based on the codes, medium, and programs. For instance, Stephens, Rand, and Melnick (2009) utilized six discrete frames (i.e., technical, economic, environmental, health and safety, political, and aesthetic/cultural frames) in assessing the risks and benefits associated with wind power technology in media reports. Roth (2014a) also applied differentiation analysis to compare word frequencies based on the medium and code of each function system (e.g., policy, economy, science, art, and law) by using Google Ngram Viewers. The result was then used to show differences in trends in functional differentiation across cultures and over time. In this study, differentiation analysis offers the framing for observing social changes by presenting keywords (codes) of research.

Furthermore, differentiation analysis brings rich insight into organizational communication research. For instance, Andersen and Born (2007) examined heterophonic organizations that do not have a primary link to a single perspective but have multiple logic such as law, education, politics, economy, help, and love systems. The authors showed how the heterophonic organizations that oscillate among multiple functional codes in communications initiate codified communication by using a semantic trick called shifter (i.e., a code that initiated communication or the switching point between the codes).

Meanwhile, differentiation analysis can be utilized in conjunction with other analytical frames. Schirmer and Michailakis (2011), for instance, analyzed the responsibility principle in healthcare priority-setting by deconstructing the human dignity principle based on a series of form-buildings and re-entries of the form. This form analysis is combined with differentiation analysis that depicts the different rationalities of the medical and the political system. Through the analyses, the authors disclose the responsibility principle as a political attempt to utilize medical reasoning for its purposes, which is fated to fail since politics cannot intervene in medical practice. Figure 4 outlines how the authors utilized form and differentiation analysis.

As seen in the research examples, the key to adopting differentiation analysis is to understand that the communication forms or perspectives of differently coded communication systems are incommensurable with each other because all systems are operationally closed, and no direct exchange is possible between them. Therefore, to narrow the communication gaps among different communication systems and facilitate coordination among them, the process of "translations" of language is necessary. To illustrate, for health communication to make the communication theme in organizational communication that follows the economic logic, health topics should be translated into the monetary terms (e.g., "improving the health status of employees contributes to an increase in the firm's profits"). As such, differentiation analysis has powerful applicability. It offers a useful device not only for diagnosing the problem of conflicting communications but for developing message strategies to resolve the problems. 
Figure 4: The analysis of attribution of agency and cause of disease combining form analysis with differentiation analysis

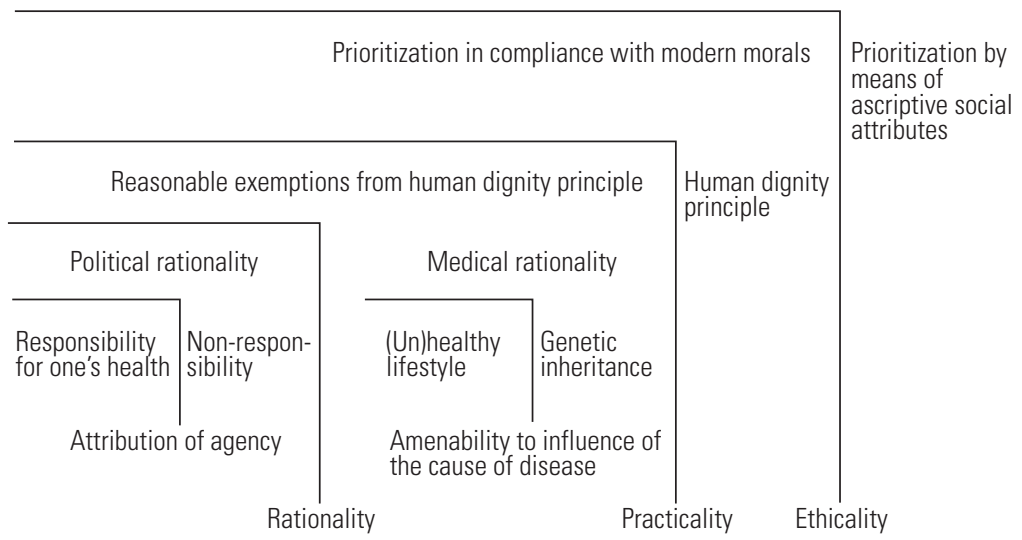

Source: Schirmer \& Michailakis, 2011, p. 277

\subsection{Semantic analysis}

Semantics. Of all types of systems, social and psychic systems are meaning-constituting systems. Psychic systems are conscious systems that have meaningful experiences, whereas social systems are communication systems that reproduce meaning by using it in communication (Luhmann, 2002/2013, p. 163). As a universal medium, meaning encompasses all. Nothing can go beyond meaning as no operation can begin without using meaning (Luhmann, 1997/2012, p. 18). Luhmann (2002/2013, p. 169) defines meaning as the medium that works with the distinction actuality/potentiality. As such, the form of meaning has two sides where one side is used at the moment of meaning being formed, while the other side remains as the potentially anticipated - i.e., the horizon of meanings. When accepting this conceptualization of meaning, meaning becomes ontologically unstable, as any moment of actualization of meaning generates new possibilities, enforcing a new actualization of meaning. Hence, meaning is viewed as the continual rearrangements of the distinction actuality/potentiality, or the constant actualizations of poten- tialities (Andersen, 2011, p. 253; Kneer \& Nassehi, 2008, p. 108).

By contrast with meaning that involves differences reactualized from moment to moment, semantics lasts longer and is considered relatively stable. Luhmann defines semantics as the "socially available sense that is generalized on a higher level and relatively independent of specific situations" (cited in Moeller, 2006, p. 51). Andersen (2011) described semantics as more "condensed and generalized forms of meaning available to communication operations", where the term condensation refers to a state in which "a multiplicity of meaning is captured in a single form, which then becomes available to an unspecified communication" (pp. 253254). By generalized forms of meaning, semantics is depicted as repeatable forms of meaning preserved for the reproduction of communication (i.e., linking communication to further communication) (Andersen \& Born, 2000, p. 304; Luhmann, $1984 / 1995$, p. 282). In a similar vein, Besio and Pronzini (2011, p. 25) described semantics as "communicative forms or distinctions which are standardized, typified and symbolized". 
Semantic analysis. As explained above, semantic analysis uses the distinction meaning / semantics as its guiding difference (Andersen, 2003, 2011). In addition, for the examination of the semantics and the assignments of meaning in society, Luhmann adopted the scheme of three meaning dimensions: factual, social, and temporal. The factual dimension involves Otherness, in "being-one-thing-and-notanother: a horse is not a cow, a number is not a pleasure, quickness not a color" (Luhmann, 1990, p. 36). The semantic of factual dimension, therefore, uses the distinction this/everything-else. The social dimension concerns what one at any time accepts as like oneself (Luhmann, 1984/1995, p. 80) and distinguishes the difference between self-reference and other-reference. Accordingly, observation of the social dimension is guided by the semantic distinction us/them. For instance, Hellenic gets its meaning in contrast with Barbarian. The temporal dimension arranges observations according to the distinction before/after or past/future. In this sense, the present is conceived as the unity of past and future. And all of the three dimensions involve distinctions with two sides.

Therefore, the working definition of semantic analysis is presented as the analysis of condensed meaning preserved for the generalized use in a society of a specific period, the analysis which has the form content/counter-content and often involves analyzing factual, social, and temporal dimensions of meaning.

Applications of semantic analysis. In social research, historically examining the semantics can offer rich insight into the society's social structures as the semantics of the society and its social structures resonate with each other, even though there is no strict causal relation between semantics and structure (Moeller, 2006, p. 51). In communication research, semantic analysis is adopted mainly to clarify the underlying and implicit distinctions constituting ideas, thoughts, symbols, and so on, that are generally accepted in a specific society.

In communication research, semantic analysis often has the form content / counter-content just like form analysis. The difference between them is that semantic analysis focuses on the condensation of meaning over time (Andersen, 2011 , p. 252), whereas form analysis focuses on analyzing the unity of distinctions or forms itself. The general questions of semantic analysis include: "How are meaning and expectations formed and how are these condensed and generalized into concepts, which then establish a semantic reservoir for systems of communication?" (Andersen, 2007, p. 124)

In communication research, semantic analysis is employed to elucidate the underlying distinctions constituting the concepts of interest and depict the nature of social phenomena. For instance, Krichewsky (2017) identified the semantic distinctions that affect defining corporate social responsibility (CSR) communication and practices, including the form ethical / unethical, substantial / window dressing, or useful / not useful.

Meanwhile, semantic analysis often adopts the aforementioned three dimensions of meanings forms: the factual, social, and temporal dimensions. The factual dimension is pertinent to the selection of themes and objects for communication; the social dimension deals with the tension between ego and alter; and the temporal dimension concerns how the tension between past and future is observed and articulated (Andersen, 2003, pp. 87-88). For instance, in analyzing CSR, Krichewsky (2017, p. 516) examined the semantic form socially responsible / irresponsible by asking which distinction refers to things (e.g., high / low levels of pollution as the factual dimension), who promotes or benefits from CSR (e.g., large companies/SMEs as the social dimension), and which comprises distinctions about time (e.g., responsibility for past events / for building a better future as the temporal dimension). Roth (2009, p. 234) also pursued the conceptualization of innovation in the organization setting by asking not just "new with respect to when?" but also "new in comparison with what?" and "new for whom?". Furthermore, the three semantic dimensions offer useful guiding categories for 
Table 1: The summary table of the four analytical frameworks

\begin{tabular}{|c|c|c|}
\hline Framework & Working Definition & Analytical Applications \\
\hline Form analysis & $\begin{array}{l}\text { The analysis of the unity of binary distinctions and their } \\
\text { re-entries for identifying the nature of social constructs } \\
\text { underlying communication and the conditions of their } \\
\text { interpretations by using the scheme of concept/ coun- } \\
\text { ter-concept or concept / context. }\end{array}$ & $\begin{array}{l}\text {-To define concepts by identifying the unity of dis- } \\
\text { tinction or performing a chain of re-entries of the } \\
\text { distinction. } \\
\text {-To capture the underlying meanings. } \\
\text {-To locate the hidden or latent communication } \\
\text { framing. } \\
\text {-To detect false distinctions. } \\
\text {-To discover emerging communication themes. } \\
\text {-To diagnose the problem in communication } \\
\text { and suggest solutions. } \\
\text {-To facilitate the sensemaking of specific concepts } \\
\text { by articulating their communicative contexts. }\end{array}$ \\
\hline Observation analysis & $\begin{array}{l}\text { The analysis of the points of reference and the conse- } \\
\text { quences of their selections based on the guiding } \\
\text { distinction observer / observed, which includes the } \\
\text { distinction self-reference / other-reference and the dis- } \\
\text { tinction second-order / first-order observation. }\end{array}$ & $\begin{array}{l}\text {-To reflect on how a choice of reference point affects } \\
\text { observation. } \\
\text {-To question what remains hidden and non-trans- } \\
\text { parent in observations. } \\
\text {-To identify conditions that influence the perceptions } \\
\text { of observers. } \\
\text {-To understand how messages are created, present- } \\
\text { ed, and interpreted in specific ways. } \\
\text {-To build strategies for message construction } \\
\text { and delivery. } \\
\text {-To diagnose and / or resolve conflicts in communi- } \\
\text { cation. }\end{array}$ \\
\hline Differentiation analysis & $\begin{array}{l}\text { The analysis and its application of the boundary condi- } \\
\text { tions distinguishing a system from its environment } \\
\text { by using communication forms such as steering binary } \\
\text { codes, medium, and / or variant programs utilized in } \\
\text { the system. }\end{array}$ & $\begin{array}{l}\text {-To explore and compare communication forms or } \\
\text { perspectives used for differently coded communica- } \\
\text { tion systems. } \\
\text {-To provide communication frames for discourse } \\
\text { analysis or media content analysis. } \\
\text {-To trace changes in the communication modes. } \\
\text {-To locate the problems of conflicting communications } \\
\text { and develop strategies to resolve the issues. }\end{array}$ \\
\hline Semantic analysis & $\begin{array}{l}\text { The analysis of condensed meaning preserved for the } \\
\text { generalized use in a society of a specific period, the } \\
\text { analysis which has the form content / counter-content } \\
\text { and often involves analyzing factual, social, and tempo- } \\
\text { ral dimensions of meaning. }\end{array}$ & $\begin{array}{l}\text {-To clarify the underlying and implicit distinctions con- } \\
\text { stituting meanings, ideas, thoughts, concepts, etc. } \\
\text {-To provide useful guiding categories for empirical } \\
\text { research. } \\
\text {-To depict the nature of social or communicative } \\
\text { phenomena. }\end{array}$ \\
\hline
\end{tabular}

empirical research. For example, Kasper, Meyer, and Schmidt (2005) explored the perceptions of work-life-balance through interviews by asking: which issues are reported (factuality), which "social others" do appear in the interviews and how are they and their expectations described (sociality), and how do the interviewees characterize their professional and private past and future and what about their time available for professional and private affairs (temporality)?

The summary of the working definitions of Luhmann's four analytical frames and their analytical applications in research is presented in Table 1.

\subsection{All-in-one: An integrated model}

Since the four analytical frameworks share the same worldview, they are conceptually intertwined so tightly that sometimes it is not clear where to draw a clear-cut border between them. Therefore, for a better understanding of the relations between them, this paper adopts the scheme of factual, social, and temporal dimensions as a heuristic tool and proposes a relationship model. ${ }^{11}$ In this model, form analysis

11 For the definitions of factual, social, and temporal dimensions, see the section on "semantic analysis". In Luhmann's original work, the object dimension is related to differentiation theory, the social dimension 
Figure 5: A relationship model of Luhmann's four fundamental analytical frames

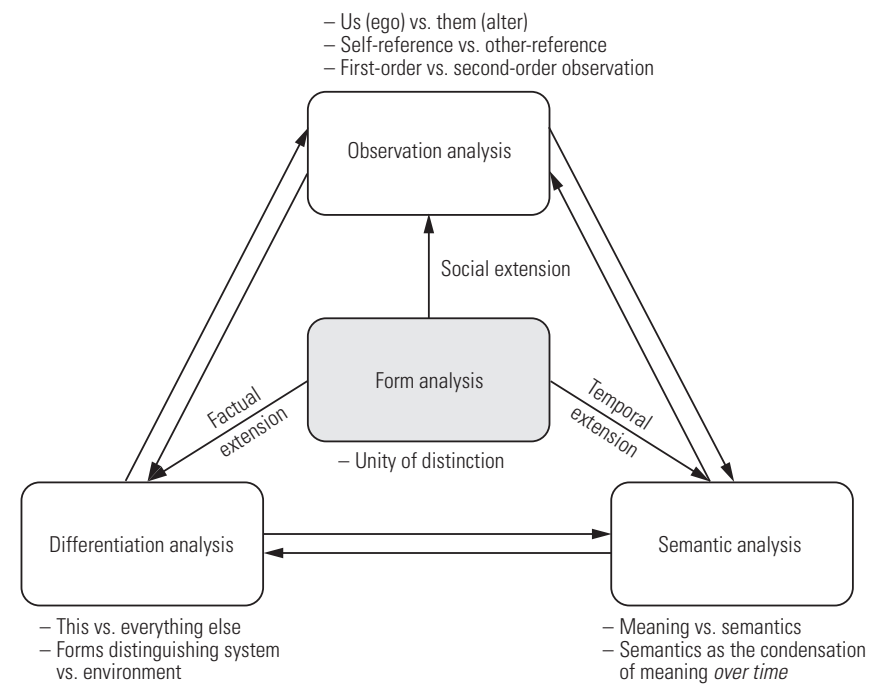

is considered the foundation on which all other frames are built and extended. That is, observation analysis is considered the social extension of form analysis. For observation analysis involves the distinction observer / observed or ego / alter and takes an observer's position as the departure point. Differentiation analysis can be described as the factual extension of form as it distinguishes a system (this) and everything else (in its environment). Finally, semantic analysis is considered the temporal extension of form analysis as it focuses on the condensation of meaning over time. This model is visualized in Figure 5.

\section{Conclusion}

By extending Andersen's (2003) propositions, the current paper formalized Luhmann's four fundamental analytical frameworks and proposed a model that

is to communication theory, and the timedimension is to social evolution theory (Luhmann, 1995). Here, the three-dimensional concepts are adopted as a heuristic tool for the explanatory purpose and not related to the original theoretical constructs. delineated the relations among them. ${ }^{12}$ In addition, to overcome the abstractness of descriptions in the existing literature, this paper suggested the workable definitions that operationalize the analytical frameworks. The exemplary communication studies presented in this paper also demonstrated the applicability or adaptability of the frameworks. Furthermore, the present paper showed the extendibility of these analytical frameworks that allows adopting them in tandem with other research methods. These research methods included - and are not limited to - case

12 Initially, Andersen (2003) developed his proposition on Luhmann's frameworks as discursive analytical strategies. The current paper prefers the term analytical frameworks to emphasize their capabilities of guiding not only conceptual discourses but also empirical research adopting diverse research methods. As demonstrated by the research examples introduced in this paper, Luhmann's analytical frameworks have been employed along with different methods such as case study, content analysis, interviews, focus groups, secondarily data analysis, etc. As to how Luhmann's analytical frameworks can be adopted in conjunction with empirical methods, Besio and Pronzini's (2011) study provides detailed guidelines. 
studies (Brier, 2006; Nobles \& Schiff, 2004; Schirmer \& Michailakis, 2011), interviews (Gregory et al., 2005; Krichewsky, 2017); focus groups (Kiisel \& Vihalemm, 2014), and field observations (Boholm, 2008).

As pointed out in the introduction, it is unfortunate that Luhmann's theory has remained marginal in the Anglophone communication scholarship. However, as demonstrated in the research examples presented in this paper, Luhmann's analytical apparatus has broad applicability and has limitless potentials for communication research. Of the vast research ideas utilizing Luhmann's analytical frameworks, for instance, one timely research area is risk communication during a pandemic.

First, form analysis can shed new light on defining pandemic communication by seeking the unity of distinction pandemic / non-pandemic and performing a chain of re-entries of this distinction. Through the re-entering, researchers can ask not only what elements form pandemic communication, but also how these factors vary contingent on the communication context. Detecting emerging communication themes by exploring core distinctions underlying pandemic communication is also possible. In particular, spotting these distinctions by searching for counter-concepts of the major terms that frequently appear in the news media will reveal the hidden media framing in pandemic communication.

Second, by utilizing observation anal$y$ sis, researchers can examine what reference points people use to describe their perceptions or experiences of a pandemic, what contexts affect their choices of reference points, and what consequences the selection of references have in thematizing the pandemic communication. In particular, the communication of the first-order observers (those who are affected by the pandemic) and that of the second-order observer (politicians and health decisionmakers) can be compared in terms of their selections of communication scopes, information channels, thematizations, the level of message complexities in communication, etc.
Third, researchers can adopt differentiation analysis to detect how the communication themes and framings of pandemic descriptions, for instance, differ by functional subsystems such as economy, politics, law, education, religion, and mass media. Also, researchers can explore translation processes that facilitate communications between these different function systems to develop effective intervention messages.

Finally, researchers employing a semantic analysis can ask what semantics repeatedly appear in pandemic communication. Research can ask several questions. In the temporal level, which future risks have formed the communication themes in the present, and which past semantics have been re-entered in the current pandemic communication? In the factual lev$e l$, which factual elements are highlighted to form pandemic communication, while other semantics remain ignored, and why? In the social level, how does the semantics of those who are affected and that of the decision-makers differ from each other? How do the risks attributed to self (or selves) differ from those to others, and what consequences of such attributions have for semantics?

To conclude, the current paper is dedicated to expanding Luhmannian research circles by providing practical and fruitful frameworks and by emphasizing their analytical gains. These theory-driven analytical frameworks are expected to provide valuable heuristic resources for those who seek to improve the consistency between theoretical constructs and methodological observations, either conceptual or empirical, thereby enriching the communication research field. In turn, more empirical applications of these frameworks will contribute to Luhmann's systems theory itself by bringing in rich insights.

\section{Conflict of interests}

The author declares no conflict of interests. 


\section{References}

Andersen, N. Å. (2003). Discursive analytical strategies: Understanding Foucault, Koselleck, Laclau, Luhmann. Bristol, UK: Policy Press.

Andersen, N. A. (2007). Creating the client who can create himself and his own fate: The tragedy of the citizens' contract. Qualitative Sociology Review, 3(2), 119-143.

Andersen, N. A. (2011). Conceptual history and the diagnostics of the present. Management \& Organizational History, 6(3), 248-267. https://doi. org/10.1177/1744935911406152

Andersen, N. Å., \& Born, A. W. (2000). Complexity and change: Two "semantic tricks" in the triumphant oscillating organization. Systemic Practice and Action Research, 13(3), 297-328. https://doi. org/10.1023/A:1009511026806

Andersen, N. Å., \& Born, A. W. (2007). Heterophony and the postponed organization organizing autopoietic systems. Tamara: Journal for Critical Organization Inquiry, 6(2), 176-186.

Andersen, N. Å., \& Stenner, P. (2020). Social immune mechanisms: Luhmann and potentialization technologies. Theory, Culture \& Society, 37(2), 79-103. https:// doi.org/10.1177/0263276419868768

Arnoldi, J. (2001). Niklas Luhmann: An introduction. Theory, Culture \& Society, 18(1), 1-13. https://doi. org/10.1177/02632760122051607

Baecker, D. (2006). The form of the firm. Organization, 13(1), 109-142. https://doi. org/10.1177/1350508406059644

Baralou, E., Wolf, P., \& Meissner, J. O. (2012). Bright, excellent, ignored: The contribution of Luhmann's system theory and its problem of non-connectivity to academic management research. Historical Social Research/Historische Sozialforschung, 37(4), 289-308. https://doi.org/10.12759/ hsr.37.2012.4.289-308

Baxter, H. (2013). Niklas Luhmann's theory of autopoietic legal systems. Annual Review of Law \& Social Science, 9, 167-184. https://doi.org/10.1146/annurevlawsocsci-102612-134027

Bergthaller, H., \& Schinko, C. (2011). Introduction: From national cultures to the semantics of modern society. In H. Bergthaller \& C. Schinko (Eds.), Addressing modernity: Social systems theory and U.S. cultures (pp. 5-33). Amsterdam, The Netherlands: Rodopi. https://doi. org/10.1163/9789042032583_002

Besio, C., \& Pronzini, A. (2011). Inside organizations and out. Methodological tenets for empirical research inspired by systems theory. Historical Social Research/Historische Sozialforschung, 36(1), 18-41. https:// doi.org/10.12759/hsr.36.2011.1.18-41

Bette, K.-H. (2015). Sa hoe ui seu po cheu: Ni keul la seu lu man ui che gye i lon e gi cho han yeon gu deul [System theory and sport: Studies based on N. Luhmann's systems theory] (H.-S. Song \& C. Lee, Trans.). Seoul, South Korea: Theorie Publishing.

Boholm, Å. (2008). The public meeting as a theatre of dissent: Risk and hazard in land use and environmental planning. Journal of Risk Research, 11(1-2), 119-140. https:// doi.org/10.1080/13669870701633852

Borch, C. (2011). Niklas Luhmann. New York, NY: Routledge.

Brier, S. (2006). Construction of knowledge in the mass media. Systemic problems in the post-modern power-struggle between the symbolic generalized media in the agora: The Lomborg case of environmental science and politics. Systems Research and Behavioral Science, 23(5), 667-684. https:// doi.org/10.1002/sres.793

Brier, S., Baecker, D., \& Thyssen, O. (2007). Foreword: Luhmann applied - For what? Cybernetics \& Human Knowing, 14(2-3), 5-10.

Egner, H., Schorch, M., Hitzler, S., Bergmann, J., \&Wulf, V. (2012). Communicating disaster - A case for qualitative approaches to disaster research. Zeitschrift für Soziologie, 41(3), 248-255. https://doi. org/10.1515/zfsoz-2012-0307

Görke, A. (2001). Entertainment as public communication: A systems-theoretic approach. Poetics, 29(4-5), 209-224. https:// doi.org/10.1016/S0304-422X(01)00043-2

Görke, A., \& Ruhrmann, G. (2003). Public communication between facts and fictions: On the construction of genetic risk. Public Understanding of Science, 12(3), 229-241. https://doi. org/10.1177/0963662503123002 
Gregory, J., Gibson, B., \& Robinson, P. G. (2005). Variation and change in the meaning of oral health related quality of life: A "grounded" systems approach. Social Science \& Medicine, 60(8), 1859-1868. https:// doi.org/10.1016/j.socscimed.2004.08.039

Hilt, L. T. (2016). “They don't know what it means to be a student": Inclusion and exclusion in the nexus between "global" and "local". Policy Futures in Education, 14(6), 666-686. https://doi. org/10.1177/1478210316645015

Holmström, S. (2007). Niklas Luhmann: Contingency, risk, trust and reflection. Public Relations Review, 33(3), 255-262. https:// doi.org/10.1016/j.pubrev.2007.05.003

Johnson, M. W., \& Leydesdorff, L. (2015). Beer's viable system model and Luhmann's communication theory: "Organizations" from the perspective of meta-games. Systems Research and Behavioral Science, 32(3), 266-282. https://doi.org/10.1002/ sres. 2222

Kasper, H., Meyer, M., \& Schmidt, A. (2005). Managers dealing with workfamily-conflict: An explorative analysis. Journal of Managerial Psychology, 20(5), 440-461. https://doi. org/10.1108/02683940510602978

Kiisel, M., \&Vihalemm, T. (2014). Why the transformation of the risk message is a healthy sign: A model of the reception of warning messages. Health, Risk \& Society, 16(3), 277-294. https://doi.org/10.10 80/13698575.2014.914471

Kneer, G., \& Nassehi, A. (2008). Niklas Luhmann eu lo ui cho dae: Niklas Luhmann eu sa hoe jeog che gye i lon [Niklas Luhmann's social systems theory] (S.-H. Jung, Trans.). Seoul, South Korea: Galmuri.

Krichewsky, D. (2017). CSR public policies in India's democracy: Ambiguities in the political regulation of corporate conduct. Business and Politics, 19(3), 510-547. https://doi.org/10.1017/bap.2017.2

Lee, C. (2013). The observing society of the operating society. Korean Journal of Sociology, 47(5), 35-71.

Luhmann, N. (1984/1995). Social systems (J. Bednarz Jr. \& D. Baecker, Trans.). Stanford, CA: Stanford University Press.
Luhmann, N. (1986/1989). Ecological communication (J. Bednarz Jr., Trans.). Chicago, IL: The University of Chicago Press.

Luhmann, N. (1990). Essays on self-reference. New York, NY: Columbia University Press.

Luhmann, N. (1996/2000). The reality of the mass media (K. Cross, Trans.). Stanford, CA: Stanford University Press.

Luhmann, N. (1997/2012). Theory of society, Vol I (R. Barrett, Trans.). Stanford, CA: Stanford University Press.

Luhmann, N. (1997/2013). Theory of society, Vol II (R. Barrett, Trans.). Stanford, CA: Stanford University Press.

Luhmann, N. (2002/2013). Introduction to systems theory (P. Gilgen, Trans. D. Baecker Ed.). Malden, MA: Polity Press.

Luhmann, N. (2005). Risk: A sociological theory. New Brunswick, NJ: Aldine Transaction.

Merton, R. K. (1959). "Notes on problem-finding in sociology”. In R. K. Merton,

L. Broom, \& L. S. Cottrell Jr. (Eds.), Sociology today: Problems and perspectives (pp. 9-34). New York, NY: Basic Books.

Michailakis, D., \& Schirmer, W. (2014). Social work and social problems: A contribution from systems theory and constructionism. International Journal of Social Welfare, 23(4), 431-442. https://doi.org/10.1111/ ijsw.12091

Moeller, H.-G. (2006). Luhmann explained: From souls to systems. Chicago, IL: Open Court Publishing.

Moeller, H.-G. (2012). The radical Luhmann. New York, NY: Columbia University Press.

Nobles, R., \& Schiff, D. (2004). A story of miscarriage: Law in the media. Journal of Law and Society, 31(2), 221-244. https://doi. org/10.1111/j.1467-6478.2004.00288.x

Roth, S. (2009). New for whom? Initial images from the social dimension of innovation. International Journal of Innovation and Sustainable Development, 4(4), 231-252. https://doi.org/10.1504/IJISD.2009.033080

Roth, S. (2011). Les deux angleterres et le continent. Anglophone sociology as the guardian of old European semantics. Journal of Sociocybernetics, 9(1-2), 19-34. https:// doi.org/10.26754/ojs_jos/jos.20111/2616

Roth, S. (2014a). Fashionable functions: A Google ngram view of trends in functional differentiation (1800-2000). International Journal of Technology and Human Interac- 
tion, 10(2), 35-58. https://doi.org/10.4018/ ijthi.2014040103

Roth, S. (2014b). The multifunctional organization: Two cases for a critical update for research programs in management and organization. Tamara: Journal for Critical Organization Inquiry, 12(3), 37-54.

Roth, S., Melkonyan, A., Kaivo-Oja, J., Manke, B., \& Dana, L. P. (2018). Interfunctional business models: Map grid for an uncharted quadrant of the blue ocean. International Journal of Entrepreneurial Venturing, 10(5), 581-595. https://doi. org/10.1504/IJEV.2018.094628

Roth, S., \& Schütz, A. (2015). Ten systems: Toward a canon of function systems. Cybernetics and Human Knowing, 22(4), 11-31. https://doi.org/10.2139/ssrn.2508950

Roth, S., Valentinov, V., \& Clausen, L. (2020). Dissecting the empirical-normative divide in business ethics: The contribution of systems theory. Sustainability Accounting, Management and Policy Journal, 11(4), 679-694. https://doi.org/10.1108/ SAMPJ-03-2019-0107

Schirmer, W., \& Michailakis, D. (2011). The responsibility principle. Contradictions of priority-setting in Swedish healthcare. Acta Sociologica, 54(3), 267-282. https:// doi.org/10.1177/0001699311412624
Sciulli, D. (1994). An interview with Niklas Luhmann. Theory, Culture \& Society, 11(2), 37-68. https://doi. org/10.1177/026327694011002003

Seidl, D. (2007). The dark side of knowledge. Emergence: Complexity \& Organization, 9(3), 16-29.

Sohn, Y. (2020). 40 years of Luhmann's legacy in the Anglophone academic community: A quantitative content analysis of Luhmannian research. International Review of Sociology, 30(3), 469-495. https://doi.org/ 10.1080/03906701.2020.1853005

Spencer-Brown, G. (1969). Laws of form. London, UK: Allen \& Unwin.

Stephens, J. C., Rand, G. M., \& Melnick, L. L. (2009). Wind energy in US media: A comparative state-level analysis of a critical climate change mitigation technology. Environmental Communication, 3(2), 168-190. https://doi. org/10.1080/17524030902916640

Šubrt, J. (2019). Niklas Luhmann's system theory: A critical analysis. RUDN Journal of Sociology, 19(4), 607-616. https://doi. org/10.22363/2313-2272-2019-19-4-607616 